\title{
Prescription de la multiplicité des valeurs propres du laplacien de Hodge-de Rham
}

Pierre Jammes

Résumé. Sur toute variété compacte de dimension supérieure ou égale à 6 , on prescrit le volume et le début du spectre du laplacien de Hodge-de Rham agissant sur les $p$-formes différentielles pour $1 \leq p<\frac{n}{2}$. En particulier, on prescrit la multiplicité des premières valeurs propres.

Abstract. On any compact manifold of dimension greater than 6, we prescribe the volume and any finite part of the spectrum of the Hodge Laplacian acting on $p$-form for $1 \leq p<\frac{n}{2}$. In particular, we prescribe the multiplicity of the first eigenvalues.

Classification mathématique par sujets (2010). 58J50.

Mots-clefs. Laplacien de Hodge-de Rham, formes différentielles, multiplicité de valeurs propres.

Keywords. Hodge Laplacian, differential forms, multiplicity of eigenvalues.

\section{Introduction}

On sait depuis les travaux de S. Y. Cheng [Ch76] que la multiplicité de la $k$-ième valeur propre du laplacien sur une surface compacte est majorée en fonction de $k$ et de la topologie. En dimension plus grande, Y. Colin de Verdière a montré ([CdV86], [CdV87]) que toute rigidité disparaît et qu'on peut arbitrairement prescrire le début du spectre, en particulier la multiplicité des valeurs propres peut être arbitrairement grande.

Le résultat de Cheng s'étend aux opérateurs de Schrödinger sur les surfaces et la majoration de la multiplicité a été améliorée (voir [Be80], [Na88], [HHN99]), la meilleure estimation pour la multiplicité de la $2^{\mathrm{e}}$ valeur propre d'un opérateur de Schrödinger sur une surface ayant été obtenue par B. Sévennec ([Sé94], [Sé02]). Ce problème a aussi été étudié pour des opérateurs avec champ magnétique ([CdVT93], [BCC98], [Er02]), pour lesquels la multiplicité peut être arbitrairement grande. En dimension supérieure ou égale à 3, J. Lohkamp a amélioré les résultats de Colin de Verdière en montrant dans [Lo96] qu' on pouvait prescrire simultanément le début du spectre, le volume et certains invariants de courbure. 
En comparaison, les connaissances sont beaucoup plus limitées concernant les opérateurs agissant sur les fibrés vectoriels naturels. P. Guérini a montré dans [Gu04] qu' on peut prescrire toute partie finie du spectre du laplacien de Hodge-de Rham, qui agit sur les formes différentielles, mais en imposant aux valeurs propres prescrites d'être simples. Un résultat semblable a été obtenu par M. Dahl pour l'opérateur de Dirac ([Da05]). Le seul résultat connu concernant la multiplicité des valeurs propres non nulles de ces deux opérateurs est qu'on peut construire un nombre arbitraire de valeurs propres doubles du laplacien de Hodge-de Rham (voir [Ja09a]). On peut consulter [Ja09b] pour une présentation générale de ces résultats.

Le but de cet article est d'étendre le théorème de Colin de Verdière aux formes différentielles en montrant que sur toute variété compacte de dimension $n \geq 6$, on peut construire des valeurs propres du laplacien de Hodge-de Rham de multiplicité arbitrairement grande, et plus précisément que si on excepte les formes de degré $\frac{n}{2}$, on peut prescrire arbitrairement le début du spectre, avec multiplicité.

Si $\left(M^{n}, g\right)$ est une variété riemannienne compacte orientable de dimension $n$, le laplacien $\Delta^{p}$ agissant sur l'espace $\Omega^{p}(M)$ des $p$-formes différentielles est défini par $\Delta=\mathrm{d} \delta+\delta \mathrm{d}$ où $\delta$ désigne la codifférentielle. Nous noterons son spectre

$$
0=\lambda_{p, 0}(M, g)<\lambda_{p, 1}(M, g) \leq \lambda_{p, 2}(M, g) \leq \cdots
$$

où les valeurs propres non nulles sont répétées s'il y a multiplicité. La multiplicité de la valeur propre nulle, si elle existe, est un invariant topologique : c'est le nombre de Betti $b_{p}(M)$. Par théorie de Hodge, le spectre $\left(\lambda_{p, i}(M, g)\right)_{i \geq 1}$ est la réunion de $\left(\mu_{p, i}(M, g)\right)_{i}$ et $\left(\mu_{p-1, i}(M, g)\right)_{i}$ où

$$
0<\mu_{p, 1}(M, g) \leq \mu_{p, 2}(M, g) \leq \cdots
$$

désigne les valeurs propres du laplacien restreint à l'espace des $p$-formes coexactes, et on a en outre $\mu_{p, i}(M, g)=\mu_{n-p-1, i}(M, g)$ pour tout $p$ et $i$ si $M$ n'a pas de bord. Le spectre complet du laplacien se déduit alors des $\mu_{p, i}(M, g)$ pour $p \leq \frac{n-1}{2}$, c'est donc à la multiplicité de ces valeurs propres qu'on va s'intéresser.

Théorème 1. Soit $M^{n}$ une variété compacte connexe orientable sans bord de dimension $n \geq 6$ et $N \in \mathbb{N}^{*}$. Si on se donne un réel $V>0$, une suite $0<a_{1,1}<a_{1,2} \leq$ $a_{1,3} \leq \cdots \leq a_{1, N}$ et des suites $0<a_{p, 1} \leq a_{p, 2} \leq \cdots \leq a_{p, N}$ pour $2 \leq p \leq\left[\frac{n-3}{2}\right]$, alors il existe une métrique $g$ sur $M$ telle que

- $\mu_{p, k}(M, g)=a_{p, k}$ pour $1 \leq k \leq N$ et $1 \leq p \leq\left[\frac{n-3}{2}\right]$;

- $\mu_{\left[\frac{n-1}{2}\right], 1}(M, g)>\sup _{i, N} a_{p, i}$;

$-\operatorname{Vol}(M, g)=V$.

Remarque 2. La condition $\mu_{\left[\frac{n-1}{2}\right], 1}(M, g)>\sup _{i, N} a_{p, i}$ permet de prescrire le début du spectre en degré $\left[\frac{n-1}{2}\right]$, les formes propres correspondantes étant exactes. 
Comme dans [CdV86] et [CdV87], le principe de la démonstration consiste à faire converger le spectre de la variété vers celui d'un espace modèle (en l'occurence un domaine de la variété) qui a le spectre souhaité et de conclure grâce aux propriétés de stabilité du spectre du modèle. Pour les formes de degrés proches de $\frac{n}{2}$, la démonstration échoue en raison de l'invariance (ou la presque invariance) conforme de la norme $L^{2}$. On verra cependant que les rigidités qui apparaissent pour ces degrés faciliteront la construction des espaces modèles. Une autre difficulté apparaît pour les formes de degré 1, on ne pourra pas faire appel aux même espaces modèles que pour les autres degrés. On utilisera une construction particulière qui ne permet pas de prescrire la multiplicité de la première valeur propre et qui ne fonctionne qu'en dimension $n \geq 6$.

Le problème suivant reste donc ouvert :

Question 3. La multiplicité des valeurs propres $\mu_{1,1}(M, g)$ et $\mu_{\left[\frac{n-1}{2}\right], k}(M, g)$ peutelle être arbitrairement grande?

Dans [Ja09a], on montre comment construire des exemples de variétés de dimension $n \geq 4$ admettant des valeurs propres de multiplicité arbitrairement grande, y compris en degré $\left[\frac{n-1}{2}\right]$. Leur topologie est très particulière (variétés produits), mais ces exemples montrent qu'on n'a pas en général de borne sur la multiplicité comme en dimension 2 .

En ce qui concerne $\mu_{1,1}(M, g)$, on peut aussi apporter cet élément de réponse en utilisant les même techniques que pour le théorème 1 :

Théorème 4. Si $M^{n}$ une variété compacte connexe orientable sans bord de dimension $n \geq 5$, alors il existe sur $M$ une métrique $g$ telle que la multiplicité de $\mu_{1,1}(M, g)$ soit égale à 3 .

La méthode utilisée ne permet cependant pas de prescrire les autres valeurs propres.

Le problème le plus intéressant semble être de comprendre ce qui se passe en dimension 3. En effet, les exemples produits donnés dans [Ja09a] sont de dimension au moins 4. L'énoncé de S. Y. Cheng pourrait s'étendre aux 1-formes coexactes en dimension 3 :

Question 5. Sur une variété $M$ de dimension 3, existe-t-il une borne sur la multiplicité de $\mu_{1, k}(M, g)$ dépendant uniquement de $k$ et de la topologie de $M$ ?

Il faut noter que pour établir un tel résultat, on peut difficilement espérer adapter la démonstration de Cheng dont les arguments sont spécifiques aux fonctions (domaines nodaux) et à la topologie en dimension 2 . 
Dans la section 2 nous montrerons, après quelques rappels techniques, qu'on peut faire tendre le spectre d'une variété compacte pour le laplacien de Hodge-de Rham vers le spectre d'un de ses domaines. La section 3 sera consacrée à la démonstration des théorèmes 1 et 4.

\section{Convergence du spectre d'une variété vers celui d'un domaine}

2.1. Conditions de bord et cohomologie pour une variété à bord. Dans ce paragraphe et le suivant, nous allons rappeler certains aspects techniques de la théorie spectrale du laplacien de Hodge-de Rham auquels nous feront appel pour montrer qu' on peut faire tendre le spectre d'une variété compacte vers celui d'un de ses domaines (théorème 11).

Si $U$ est un domaine à bord $C^{1}$ d'une variété compacte $M$, on note $j: \partial U \rightarrow \bar{U}$ l'injection canonique et $N$ un champ de vecteur normal au bord. Il existe plusieurs conditions de bord admissibles pour le laplacien de Hodge-de Rham sur $\bar{U}$ (c'està-dire telles que le laplacien soit elliptique), les deux principales sont les conditions absolues et relatives, que nous noterons respectivement (A) et (R) et qui sont définies par

$$
\text { (A) }\left\{\begin{array} { l } 
{ j ^ { * } ( \iota _ { N } \omega ) = 0 , } \\
{ j ^ { * } ( \iota _ { N } \mathrm { d } \omega ) = 0 , }
\end{array} \text { ou } \left\{\begin{array}{l}
j^{*}(* \omega)=0 \\
j^{*}(* \mathrm{~d} \omega)=0
\end{array}\right.\right.
$$

et

$$
\text { (R) }\left\{\begin{array}{l}
j^{*}(\omega)=0, \\
j^{*}(\omega)=0 .
\end{array}\right.
$$

Pour la condition (A), Ker $\Delta$ est isomorphe à la cohomologie $H^{p}(U)$ et pour $(\mathrm{R})$, il est isomorphe à la cohomologie à support compact $H_{0}^{p}(U)$ (voir par exemple [Ta96], ch. 5). Rappelons aussi que les cohomologies de $U$ et $\bar{U}$ sont isomorphes ([Ta96], ch. 5, p. 375). Il est immédiat que sous la condition (R) on a $j^{*}(\mathrm{~d} \omega)=0$. Et comme la dualité de Hodge permute ces deux conditions de bord, (A) implique que $j^{*}(* \delta \omega)=0$.

Nous aurons besoin d'une autre condition de bord, définie par

$$
\text { (D) }\left\{\begin{array}{l}
j^{*}(\omega)=0 \\
j^{*}(* \omega)=0
\end{array}\right.
$$

Pour la condition (D), le noyau du laplacien est trivial (voir [An89]).

Rappelons qu'en restriction aux fonctions, la condition (A) est équivalente à la condition de Neumann, et les conditions (R) et (D) à la condition de Dirichlet.

La décomposition de Hodge $L^{2}\left(\Lambda^{p} \bar{U}\right)=\operatorname{Im} \mathrm{d} \oplus \operatorname{Ker} \Delta \oplus \operatorname{Im} \delta$ dépend de la condition de bord choisie. Pour une forme $\omega$, elle s'écrit $\omega=\mathrm{d} \delta \theta+\alpha+\delta \mathrm{d} \theta$ où $\alpha$ et 
$\theta$ vérifie la condition de bord considérée, $\alpha$ étant dans le noyau Ker $\Delta$ correspondant (cf. proposition 9.8 de [Ta96]).

Dans la suite du texte interviendra essentiellement la condition (A) (et dans une moindre mesure la condition (D)), et nous ferons en particulier appel à la propriété suivante :

Proposition 6. Si $\omega$ est une p-forme exacte sur $\bar{U}$, alors il existe une forme $\theta \in$ $\Omega^{p}(\bar{U})$ vérifiant la condition (A) et telle que $\omega=\mathrm{d} \delta \theta$. En particulier, la forme $\varphi=\delta \theta$ vérifie $j^{*}\left(\mathrm{l}_{N} \varphi\right)=0$, est orthogonale aux formes fermées et minimise la norme $L^{2}$ parmi les primitives de $\omega$.

La démonstration de ces faits (qui ne sont souvent utilisés qu'implicitement dans la littérature) est esquissée dans [Ch79] (section 3, p. 272). Nous allons la rappeler :

Démonstration. Si $\alpha \in \Omega^{p}(\bar{U})$ et $\beta \in \Omega^{p+1}(\bar{U})$, une intégration par partie donne $(\mathrm{d} \alpha, \beta)=(\alpha, \delta \beta)+\int_{\partial U} j^{*}(\alpha \wedge * \beta)$. Pour que le terme de bord s'annule, il n'est pas nécessaire que les deux formes $\alpha$ et $\beta$ vérifient l'une des conditions de bord, il suffit par exemple que $j^{*}(* \beta)=0$, c'est-à-dire que $\beta$ soit tangentielle.

Si $\omega$ est une forme exacte, on a alors $(\omega, \beta)=0$ pour toute forme $\beta$ tangentielle cofermée. Dans la décomposition de Hodge pour la condition (A), la composante cofermée de $\omega$ est donc nulle même si $\omega$ ne vérifie aucune condition de bord. On a donc $\omega=\mathrm{d} \delta \theta$ où $\theta$ vérifie la condition (A) (autrement dit, l'adhérence $L^{2}$ des formes exactes vérifiant (A) contient toutes les formes exactes sur $\bar{U}$, sans condition de bord).

Si on pose $\varphi=\delta \theta$, on a alors $j^{*}\left(\mathrm{l}_{N} \varphi\right)=0$, et $(\alpha, \varphi)=(\mathrm{d} \alpha, \theta)=0$ pour toute forme $\alpha$ fermée. Comme deux primitives de $\omega$ diffèrent par une forme fermée, $\varphi$ minimise la norme $L^{2}$ parmi celles-ci.

Nous aurons besoin de définir, outre les cohomologies $H^{p}(U)$ et $H_{0}^{p}(U)$ évoquées plus haut, un espace de cohomologie traduisant l'interaction entre la cohomologie de $U$ et celle de $M$. Cet espace est construit comme le quotient des formes fermées de $\bar{U}$ par la restriction des formes fermées de $M$ :

$$
H^{p}(U / M)=\left\{\omega \in \Omega^{p}(\bar{U}), \mathrm{d} \omega=0\right\} /\left\{\omega_{\mid \bar{U}}, \omega \in \Omega^{p}(M) \text { et } \mathrm{d} \omega=0\right\}
$$

Comme une forme exacte de $\bar{U}$ est toujours la restriction d'une forme exacte de $M, H^{p}(U / M)$ est isomorphe au quotient de $H^{p}(\bar{U}) \simeq H^{p}(U)$ par l'image de l'application naturelle $H^{p}(M) \rightarrow H^{p}(\bar{U})$ définie par restriction des formes fermées et exactes. En particulier, $H^{p}(U / M)$ est de dimension finie.

2.2. Caractérisation du spectre du laplacien de Hodge. Pour démontrer le théorème de convergence au paragraphe suivant, nous utiliserons une caractérisation variationnelle du spectre dont le principe est dû à J. Cheeger et J. Dodziuk : 
Proposition 7 ([Do82], [Mc93]). Sur une variété compacte sans bord ou avec condition de bord (A), on a

$$
\mu_{p, i}=\inf _{V_{i}} \sup _{\omega \in V_{i} \backslash\{0\}}\left\{\frac{\|\omega\|^{2}}{\|\varphi\|^{2}}, \mathrm{~d} \varphi=\omega\right\},
$$

où $V_{i}$ parcourt l'ensemble des sous-espaces de dimension $i$ dans l'espace des $p+1$ formes exactes lisses.

Il faut préciser que dans le cas à bord, cette formule fournit le spectre pour la condition (A) même si on ne suppose pas que des formes $\omega$ et $\varphi$ vérifient cette condition. Cela tient essentiellement au fait démontré plus haut que l'espace $L^{2}$ des formes exactes pour la décomposition de Hodge associée à (A) contient toutes les formes exactes sans condition de bord.

La proposition 7 permet d'estimer les valeurs propres du laplacien, mais pour les applications à la multiplicité, et en particulier pour utiliser les techniques de Colin de Verdière (voir lemmes 16 et 17), nous aurons besoin de contrôler à la fois les valeurs propres et les espaces propres, nous allons donc la reformuler. $\mathrm{Si} \omega$ est une forme exacte, alors $q(\omega)=\inf _{\mathrm{d} \varphi=\omega}\|\varphi\|^{2}$ est une forme quadratique, c'est la norme au carré de la primitive coexacte de $\omega$. Son spectre est l'inverse de celui du laplacien (on peut se convaincre qu'on a aussi $q(\omega)=\left(\Delta^{-1} \omega, \omega\right)$ ). On retrouve la formule de la proposition 7 en intervertissant le rôle de la forme quadratique et de la norme de Hilbert :

Proposition 8. Le spectre et les espaces propres du laplacien en restriction aux formes exactes sont ceux de la forme quadratique $Q(\omega)=\|\omega\|_{L^{2}}^{2}$ relativement à la norme $|\omega|=\inf _{\mathrm{d} \varphi=\omega}\|\varphi\|_{L^{2}}$.

Remarque 9. L'espace $\overline{L^{2}\left(\Lambda^{p+1} M\right) \cap \operatorname{Im} \mathrm{d}}$ (l'adhérence étant prise au sens de la norme $L^{2}$ ) n'est pas un espace de Hilbert car il n'est pas complet pour la norme $|\cdot|$ (c'est seulement le domaine de la forme quadratique $Q$ ), mais on peut identifier son complété à $L^{2}\left(\Lambda^{p} M\right) / \overline{\text { Ker d }}$, chaque forme exacte de $L^{2}\left(\Lambda^{p+1} M\right)$ étant identifiée à l'ensemble de ses primitives.

Remarque 10. On peut déduire aisément de la proposition 7 que le spectre du laplacien est continu pour la topologie $C^{0}$ (cf. [Do82]). On sait aussi, d'après [BD97] qu' on a continuité des espaces propres pour les topologies $C^{0}$ et $L^{2}$. La formulation de la proposition 8 permet de retrouver ce fait de manière plus directe à l'aide des techniques de [CdV86] (voir le lemme 17 au paragraphe suivant).

2.3. Convergence spectrale. Nous allons maintenant montrer comment on peut faire tendre le spectre du laplacien de Hodge-de Rham d'une variété compacte vers 
celui d'un domaine, généralisant ainsi au formes différentielles le théorème que Y. Colin de Verdière a montré pour les fonctions (théorème III.1 de [CdV86]). Pour appliquer ce résultat, nous aurons besoin d'une certaine uniformité de la convergence, nous reprendrons pour cela les notations de [CdV86] :

Soit $E_{0}$ et $E_{1}$ sont deux sous-espaces vectoriels de même dimension $N$ d'un espace de Hilbert, munis respectivement des formes quadratiques $q_{0}$ et $q_{1}$. Si $E_{0}$ et $E_{1}$ sont suffisamment proches, il existe une isométrie naturelle $\psi$ entre les deux (voir la section I de [CdV86] pour les détails de la construction), on définit alors l'écart entre $q_{0}$ et $q_{1}$ par $\left\|q_{1} \circ \psi-q_{0}\right\|$. Pour deux formes quadratiques $Q_{0}$ et $Q_{1}$ sur l'espace de Hilbert, on appellera $N$-écart spectral entre $Q_{0}$ et $Q_{1}$ l'écart entre les deux formes quadratiques restreintes à la somme des espaces propres associés aux $N$ premières valeurs propres. Si cet écart est petit, alors les $N$ premières valeurs propres de $Q_{0}$ et leurs espaces propres sont proches de ceux de $Q_{1}$.

On veut montrer que la convergence spectrale est uniforme pour une certaine famille de spectres limites. Comme dans [CdV86] on dira donc qu'une forme quadratique vérifie l'hypothèse $(*)$ si ses valeurs propres vérifient

$$
\lambda_{1} \leq \cdots \leq \lambda_{N}<\lambda_{N}+\eta \leq \lambda_{N+1} \leq M
$$

pour un entier $N$ et des réels $\eta, M>0$ fixés une fois pour toute.

Dans la suite du texte, sauf mention explicitement contraire, le spectre considéré sur les domaines sera toujours relatif aux conditions de bord (A).

Théorème 11. Soit $\left(M^{n}, g\right)$ une variété riemanienne compacte sans bord de dimension $n$ et $U$ un domaine de $M$ à bord $C^{1}$. Il existe une suite de métriques $\left(g_{i}\right)$ sur $M$ conformes à $g$ telle que

$-\operatorname{Vol}\left(M, g_{i}\right) \rightarrow \operatorname{Vol}(U, g)$ quand $i \rightarrow \infty$;

- $\mu_{p, k}\left(M, g_{i}\right) \rightarrow 0$ pour $p \leq \frac{[n-3]}{2}$ et $k \leq d_{p}$ quand $i \rightarrow \infty$;

- $\mu_{p, k+d_{p}}\left(M, g_{i}\right) \rightarrow \mu_{p, k}(U, g)$ pour $p \leq \frac{[n-3]}{2}$ et $k \geq 1$ quand $i \rightarrow \infty$; où $d_{p}$ est la dimension de $H^{p}(U / M)$.

En outre, si les $\mu_{p, k}(U, g)$ vérifient l'hypothèse $(*)$ pour $1 \leq p \leq \frac{[n-3]}{2}$, alors pour tout $\varepsilon>0$ il existe $i$ tel que le $N$-écart spectral entre les laplaciens sur $U$ et $M$ pour la métrique $g_{i}$ soit inférieur à $\varepsilon$.

Remarque 12. Dans [Co04], B. Colbois avait posé la question de savoir si on peut faire tendre les valeurs propres du laplacien de Hodge-de Rham vers 0 en fixant le volume et la classe conforme. Pour $p \leq \frac{[n-3]}{2}$, une réponse positive a été donnée dans [Ja08] par une constrution similaire à celle du théorème 11 avec $U \simeq S^{p} \times B^{n-p}$. Le théorème 11 permet une compréhension plus générale de ce phénomène, tout en simplifiant les démonstrations de [Ja08].

On sait que l'énoncé du théorème 11 est faux pour $p=\left[\frac{n-1}{2}\right]$, car pour ce degré, le spectre du laplacien est uniformément minoré dans une classe conforme : 
Théorème 13 ([Ja07]). Soit $M^{n}$ une variété compacte de dimension $n \geq 3$, Pour toute classe conforme $C$ sur $M$, il existe une constante $K(C)>0$ telle que pour toute métrique $g \in C$, on a

$$
\mu_{\left[\frac{n-1}{2}\right], 1}(M, g) \operatorname{Vol}(M, g)^{\frac{2}{n}} \geq K .
$$

On peut donc construire des contre-exemples en choissant $M$ et $U$ tels que $H^{\left[\frac{n-1}{2}\right]}(M / U)$ soit non trivial, ou en utilisant un domaine dont la première valeur propre est plus petite que que $K \cdot \operatorname{Vol}(U, g)^{-\frac{2}{n}}$ (c'est possible d'après [Gu04]).

Remarque 14. La constante $K$ du théorème 13 varie continûment pour des déformations $C^{0}$ de la métrique (voir [Ja07]). Cette propriété nous sera utile pour démontrer le théorème 1 .

Remarque 15. Pour l'opérateur de Dirac, il existe une rigidité conforme du même type que celle du théorème 13 (voir [Am03]). On ne peut donc pas espérer obtenir un résultat semblable au théorème 11 pour les spineurs.

Comme dans [CdV86], on fera appel aux deux lemmes qui suivent. Les constantes $N, M$ et $\eta$ qui interviennent dans les énoncés font référence à l'hypothèse (*) définie plus haut.

Lemme 16 ([CdV86], th. I.7). Soit $Q$ une forme quadratique positive sur un espace de Hilbert $\mathscr{H}$ dont le domaine admet la décomposition $Q$-orthogonale $\operatorname{dom}(Q)=$ $\mathscr{H}_{0} \oplus \mathscr{H}_{\infty}$. Pour tout $\varepsilon>0$, il existe une constante $C(\eta, M, N, \varepsilon)>0$ (grande) telle que si $Q_{0}=Q_{\mid H_{0}}$ vérifie l'hypothèse $(*)$ et que $Q(x) \geq C|x|^{2}$ pour tout $x \in \mathscr{H}_{\infty}$, alors $Q$ et $Q_{0}$ ont un $N$-écart spectral inférieur à $\varepsilon$.

Lemme 17 ([CdV86], th. I.8). Soit ( $\mathscr{H},|\cdot|)$ un espace de Hilbert muni d'une forme quadratique positive $Q$. On se donne en outre une suite de métriques $|\cdot|_{n}$ sur $\mathscr{H}$ et une suite de formes quadratiques $Q_{n}$ de même domaine que $Q$ telles que:

(i) il existe $C_{1}, C_{2}>0$ tels que $C_{1}|x| \leq|x|_{n} \leq C_{2}|x|$ pour tout $x \in \mathscr{H}$;

(ii) pour tout $x \in \operatorname{dom}(Q),|x|_{n} \rightarrow|x|$;

(iii) pour tout $x \in \operatorname{dom}(Q), Q(x) \leq Q_{n}(x)$;

(iv) pour tout $x \in \operatorname{dom}(Q), Q_{n}(x) \rightarrow Q(x)$.

Si $Q$ vérifie l'hypothèse (*), alors à partir d'un certain rang (dépendant de $\eta$, $M$ et $N)$, $Q$ et $Q_{n}$ ont un $N$-écart spectral inférieur à $\varepsilon$.

Remarque 18. Dans le lemme 17, on peut affaiblir l'hypothèse $C_{1}|x| \leq|x|_{n} \leq$ $C_{2}|x|$ en $C_{1}|x| \leq|x|_{n} \leq C_{2}|x|+\varepsilon_{n} Q(x)^{\frac{1}{2}}$ avec $\varepsilon_{n} \rightarrow 0$, la démonstration restant exactement la même. En particulier, il n'est pas nécessaire que l'espace de Hilbert $(\mathscr{H},|\cdot|)$ soit complet pour $|\cdot|_{n}$. 
Remarque 19. Pour déduire la convergence du spectre et des espaces propres de la convergence des formes quadratiques, on doit en principe se ramener à une norme de Hilbert fixe. Ça ne sera pas nécessaire dans la suite car les étapes de la démonstration où la norme varie seront traitées à l'aide du lemme 17.

Démonstration du théorème 11. En vertu de la proposition 8, il suffit de montrer la convergence des valeurs propres et des espaces propres de la forme quadratique $Q(\omega)=\|\omega\|^{2}$ relativement à la norme $|\omega|=\inf _{\mathrm{d} \varphi=\omega}\|\varphi\|$ pour les formes différentielles exactes de degré 1 à $\left[\frac{n-1}{2}\right]$ (les $\mu_{p, i}$ sont les valeurs propres de $Q$ sur les formes exactes de degré $p+1$ ).

Comme dans [CdV86], on va passer par l'intermédiaire d'une famille de métriques singulières $\left(g_{\varepsilon}\right)_{\varepsilon \in] 0,1]}$ définies par $g_{\varepsilon}=g$ sur $U$ et $g_{\varepsilon}=\varepsilon^{2} g$ sur $M \backslash U$. Notons que la forme quadratique $Q_{\varepsilon}$ et la métrique $|\cdot|_{\varepsilon}$ associées à $g_{\varepsilon}$ sont bien définies. Plus précisément, pour une forme exacte $\omega \in \Omega^{p+1}(M)$, on a

$$
Q_{\varepsilon}(\omega)=\int_{U}|\omega|^{2} \mathrm{~d} v_{g}+\varepsilon^{n-2 p-2} \int_{M \backslash U}|\omega|^{2} \mathrm{~d} v_{g}
$$

et

$$
|\omega|_{\varepsilon}^{2}=\inf _{\mathrm{d} \varphi=\omega}\left(\int_{U}|\varphi|^{2} \mathrm{~d} v_{g}+\varepsilon^{n-2 p} \int_{M \backslash U}|\varphi|^{2} \mathrm{~d} v_{g}\right) .
$$

Pour $\varepsilon$ fixé, les normes induites par $g$ et $g_{\varepsilon}$ sont équivalentes. Il en va donc de même pour les normes $|\cdot|$ et $|\cdot|_{\varepsilon}$, ainsi que pour les normes d'opérateurs associées à $Q$ et $Q_{\varepsilon}$. Les résultats usuels de théorie spectrale (spectre formé d'une suite de valeurs propres tendant vers l'infini) s'appliquent donc à la forme quadratique $Q_{\varepsilon}$.

La démonstration se déroule en trois étapes. D'abord, on montre que pour $\varepsilon$ donné, on peut approcher la métrique $g_{\varepsilon}$ par une métrique lisse avec convergence du spectre. Comme pour tout $\varepsilon$ on peut trouver une métrique lisse dont l'écart spectral avec $g_{\varepsilon}$ soit arbitrairement petit, il suffit de montrer que l'écart spectral entre le spectre de $g_{\varepsilon}$ et celui de $U$ devient lui ausssi arbitrairement petit quand $\varepsilon \rightarrow 0$, ce qui fait l'objet de la suite de la démonstration. La deuxième étape consiste à décomposer l'espace $\mathscr{H}$ des formes exactes en une somme $\mathscr{H}_{0} \oplus \mathcal{H}_{\infty}$ à laquelle on applique le lemme 16. Enfin, on montre la convergence du spectre de $Q_{\varepsilon}$ restreint à $\mathscr{H}_{0}$ vers le spectre du domaine à l'aide du lemme 17.

Étape 1. En utilisant le lemme 17, on va montrer qu'on peut approcher $g_{\varepsilon}$ par des métriques lisses avec convergence du volume, du spectre et des espaces propres.

Pour un $\varepsilon$ fixé, on peut approcher la fonction $\chi_{U}+\varepsilon \chi_{M \backslash U}$ par une suite de fonctions décroissantes $\left(f_{j}\right)$. La suite de métriques $g_{j}=f_{j}^{2} \cdot g$ tend alors vers $g_{\varepsilon}$, et on note $Q_{j}$ et $|\cdot|_{j}$ la forme quadratique et la norme hilbertienne associées. On peut vérifier que la suite de forme quadratique $Q_{j}$ converge simplement vers $Q_{\varepsilon}$ (hypothèse (iv) du lemme 17). Comme il existe une constante $C$ telle que $g_{\varepsilon} \leq g_{j} \leq C \cdot g_{\varepsilon}$, on a $|\omega|_{\varepsilon}^{2} \leq|\omega|_{j}^{2} \leq C \cdot|\omega|_{\varepsilon}^{2}$ et $Q_{\varepsilon}(\omega) \leq Q_{j}(\omega)$ pour toute forme exacte $\omega$ (hypothèses (i) 
et (iii) du lemme). Pour appliquer le lemme 17, il reste à vérifier que l'hypothèse (ii) est satisfaite, à savoir que $|\cdot|_{j}$ converge simplement vers $|\cdot|_{\varepsilon}$. Étant donné $\eta>0$, il existe une forme $\varphi_{0}$ telle que $\mathrm{d} \varphi_{0}=\omega$ et $\left\|\varphi_{0}\right\|_{g_{\varepsilon}}^{2} \leq|\omega|_{\varepsilon}^{2}+\eta$ (par définition de $|\cdot|_{\varepsilon}$ ). Comme $g_{j}$ converge vers $g_{\varepsilon}$, pour $j$ assez grand on a aussi $\left\|\varphi_{0}\right\|_{g_{j}}^{2} \leq\left\|\varphi_{0}\right\|_{g_{\varepsilon}}^{2}+\eta$, et donc $|\omega|_{\varepsilon}^{2} \leq|\omega|_{j}^{2} \leq\left\|\varphi_{0}\right\|_{g_{j}}^{2} \leq|\omega|_{\varepsilon}^{2}+2 \eta$. On a donc bien $|\omega|_{j} \rightarrow|\omega|_{\varepsilon}$ pour tout $\omega$.

Selon le lemme 17, à $\varepsilon$ fixé, on peut donc trouver un $j_{\varepsilon}$ tel que l'écart spectral entre $Q_{j_{\varepsilon}}$ et $Q_{\varepsilon}$ soit arbitrairement petit.

Étape 2. On va décomposer l'espace des $(p+1)$-formes exactes en une somme $\mathscr{H}_{0} \oplus \mathscr{H}_{\infty}$ à laquelle on applique le lemme 16 pour la norme $|\cdot|_{\varepsilon}$ et la forme quadratique $Q_{\varepsilon}$.

On commence par définir le sous-espace $\mathscr{H}_{\infty}$ de $\overline{\operatorname{Im~d}^{p}} \subset L^{2}\left(\Lambda^{p+1} M\right)$ comme l'adhérence des différentielles des formes lisses qui s'annulent sur $U$ (les adhérences sont au sens de la norme $L^{2}$ ). Une telle forme va nécessairement vérifier la condition de bord de Dirichlet sur $M \backslash U$ :

$$
\mathscr{H}_{\infty}=\overline{\left\{\mathrm{d} \varphi, \varphi \in \Omega^{p}(M), \varphi_{\mid U}=0, \varphi_{\mid M \backslash U} \text { vérifie (D) }\right\}} .
$$

L'espace $\mathscr{H}_{0}$ sera défini comme la somme de deux espaces $\mathscr{H}_{1}$ et $\mathscr{H}_{2}$ construits séparément.

Soit $\omega$ une $(p+1)$-forme exacte sur $\bar{U}$ et $\varphi \in \Omega^{p}(\bar{U})$ la primitive coexacte de $\omega$ fournie par la proposition 6 . Si $\tilde{\varphi}$ est un prolongement lisse de $\varphi$ sur $M$, alors d $\tilde{\varphi}$ est définie à un élement de $\mathscr{H}_{\infty}$ près. On peut définir alors $\tilde{\omega}$ comme le d $\tilde{\varphi}$ de norme minimale pour $g_{\varepsilon}$. Cet infimum est bien atteint dans $L^{2}$ et on peut le construire par projection sur l'orthogonal $L^{2}$ de $\mathscr{H}_{\infty}$. On pose alors

$$
\mathscr{H}_{1}=\overline{\left\{\tilde{\omega}, \omega \in \Omega^{p+1}(\bar{U}) \text { exacte }\right\}} .
$$

L'espace $\mathscr{H}_{2}$ est défini à partir de l'espace de cohomologie $H^{p}(U / M)$. Comme $H^{p}(U / M)$ est isomorphe au quotient de $H^{p}(U)$ par le sous-espace induit par $H^{p}(M)$, il est aussi isomorphe à l'orthogonal, dans l'espace des formes harmoniques de $U$ (avec condition de bord absolue), des représentants harmoniques des classes de cohomologie induites par $H^{p}(M)$. On peut ainsi définir un représentant harmonique $h$ de chaque classe $[c] \in H^{p}(U / M)$, qui est la forme $h \in[c]$ orthogonale aux restrictions des formes fermées de $M$.

On peut alors construire $\mathscr{H}_{2}$ sur le modèle de $\mathscr{H}_{1}$. Chaque forme harmonique $h$ représentant une classe de $H^{p}(U / M)$, peut être étendue en une forme $\tilde{h}$ sur $M$, la forme $\mathrm{d} \tilde{h}$ étant alors définie à un élement de $\mathscr{H}_{\infty}$ près. On notant $\tilde{\omega}_{h}$ la forme $\mathrm{d} \tilde{h}$ qui est $L^{2}$-orthogonale à $\mathscr{H}_{\infty}$, on pose

$$
\mathscr{H}_{2}=\left\{\tilde{\omega}_{h},[h] \in H^{p}(U / M)\right\} .
$$

Par construction, $\mathscr{H}_{0}=\mathscr{H}_{1} \oplus \mathscr{H}_{2}$ et $\mathscr{H}_{\infty}$ sont orthogonaux pour la norme $L^{2}$, donc $Q$-orthogonaux. Avant d'appliquer le lemme 16 on doit encore vérifier que 
$\mathscr{H}_{0} \oplus \mathscr{H}_{\infty}$ contient bien toutes les $(p+1)$-formes exactes. Soit $\omega \in \Omega^{p+1}$ une forme exacte. Par définition de $\mathscr{H}_{1}$, on peut écrire $\omega=\omega_{1}+\omega^{\prime}$, avec $\omega_{1} \in \mathscr{H}_{1}$ telle que $\omega_{\mid \bar{U}}=\omega_{1 \mid \bar{U}}$ et $\omega_{\mid \bar{U}}^{\prime}=0$. Comme $\omega$ et $\omega_{1}$ sont exactes, $\omega^{\prime}$ l'est aussi. Si on pose $\omega^{\prime}=\mathrm{d} \varphi$, la forme $\varphi$ est définie à une forme fermée près et vérifie $\mathrm{d} \varphi=0$ sur $\bar{U}$, la classe $[\varphi] \in H^{p}(U / M)$ est donc bien définie. Par définition de $\mathscr{H}_{2}$ on a alors $\omega^{\prime}=\tilde{\omega}_{h}+\omega_{0}$, où $\tilde{\omega}_{h}$ est l'élément de $\mathscr{H}_{2}$ associé au représentant harmonique $h$ de $[\varphi] \in H^{p}(U / M)$, et $\omega_{0} \in \mathscr{H}_{\infty}$. On a donc bien $\omega \in \mathscr{H}_{1} \oplus \mathscr{H}_{2} \oplus \mathscr{H}_{\infty}$, et donc $\operatorname{dom}\left(Q_{\varepsilon}\right)=\mathscr{H}_{0} \oplus \mathscr{H}_{\infty}$.

Si on note $\lambda^{(\mathrm{D})}$ la première valeur propre de $M \backslash U$ pour la métrique $g$ et la condition de bord (D), on sait que pour toute forme $\omega \in \mathscr{H}_{\infty}$ il existe, par définition, une forme $\varphi \in \Omega^{p}(M)$ à support dans $M \backslash U$ telle que $\mathrm{d} \varphi=\omega$ et $\|\omega\|^{2} /\|\varphi\|^{2} \geq \lambda^{(\mathrm{D})}$ pour la métrique $g$. Pour la métrique $g_{\varepsilon}$, on a donc $\|\omega\|^{2} /\|\varphi\|^{2} \geq \varepsilon^{-2} \lambda^{(\mathrm{D})}$, et a fortiori $Q_{\varepsilon}(\omega) /|\omega|_{\varepsilon} \geq \varepsilon^{-2} \lambda^{(\mathrm{D})}$. Si $\varepsilon$ est suffisamment petit, on peut appliquer le lemme 16 .

Étape 3. On va achever la démonstration en appliquant le lemme 17 et la remarque 18 à l'espace $\mathscr{H}_{0}$ et aux familles de métriques et de formes quadratiques $|\cdot|_{\varepsilon}$ et $Q_{\varepsilon}$. On définit la forme quadratique $Q$ sur $\mathscr{H}_{0}$ par $Q(\omega)=\int_{U}|\omega|^{2} \mathrm{~d} v_{g}$. On doit aussi définir une norme $|\cdot|$ sur $\mathscr{H}_{0}$ : pour $\omega \in \mathscr{H}_{1}$, on note $\varphi_{\omega}$ la primitive coexacte de $\omega_{\mid \bar{U}}$ donnée par la proposition 6, et pour $\omega \in \mathscr{H}_{2}$, on note $\varphi_{\omega}$ le représentant harmonique de la classe de cohomologie définie par $\omega$. On étend linéairement l'application $\omega \rightarrow \varphi_{\omega}$ et on pose $|\omega|=\left\|\varphi_{\omega}\right\|$, la norme $\|\cdot\|$ étant ici la norme $L^{2}$ sur les $p$-formes de $U$. Les espaces $\mathscr{H}_{1}$ et $\mathscr{H}_{2}$ sont orthogonaux pour $\|\cdot\|$, le noyau de la forme quadratique $Q$ (relativement à $\|\cdot\|$ ) est $\mathscr{H}_{2}$, et le spectre de $Q$ sur $\mathscr{H}_{1}$ est le spectre du domaine $U$ pour les $(p+1)$-formes exactes.

On doit maintenant vérifier que les quatre hypothèses du lemme 17 sont satisfaites.

Les hypothèses (iii) et (iv) sont les plus simples à vérifier : par définition de $Q_{\varepsilon}$, pour tout $\omega \in \mathscr{H}_{0}, Q_{\varepsilon}(\omega)$ tend vers $Q(\omega)=\int_{U}|\omega|^{2} \mathrm{~d} v_{g}$, c'est-à-dire la norme $L^{2}$ au carré de $\omega$ restreinte à $U$ et $Q_{\varepsilon} \geq Q$ pour tout $\varepsilon$.

Passons à l'hypothèse (ii). Par définition de $\mathscr{H}_{1}$ et $\mathscr{H}_{2}$, il existe pour tout $\omega \in \mathscr{H}_{0}$ un prolongement $\tilde{\varphi}_{\omega}$ de $\varphi_{\omega}$ tel que $\mathrm{d} \tilde{\varphi}_{\omega}=\omega$. On a alors

$$
|\omega|_{\varepsilon}^{2} \leq\left\|\tilde{\varphi}_{\omega}\right\|_{\varepsilon}^{2} \rightarrow \int_{U}\left|\tilde{\varphi}_{\omega}\right|^{2} \mathrm{~d} v_{g}=|\omega|^{2} .
$$

En outre, si $\mathrm{d} \varphi=\omega$, alors $\varphi$ et $\tilde{\varphi}_{\omega}$ ne diffèrent que par une forme fermée de $M$, et donc leur restriction à $\bar{U}$ ne diffèrent aussi que par une forme fermée. Par conséquent, la norme de $\varphi_{\omega}$ minore la norme sur $U$ de $\varphi$, et

$$
|\omega|^{2}=\int_{U}\left|\tilde{\varphi}_{\omega}\right|^{2} \mathrm{~d} v_{g} \leq \int_{U}|\varphi|^{2} \mathrm{~d} v_{g} \leq\|\varphi\|_{\varepsilon}^{2}
$$

On en déduit que $|\omega| \leq|\omega|_{\varepsilon}$, et donc que $|\omega|_{\varepsilon} \rightarrow|\omega|$ pour tout $\omega$. 
L'hypothèse (i), dans sa version faible (cf. remarque 18), est la plus technique à vérifier. On doit contrôler $|\cdot|_{\varepsilon}$ en fonction de $|\cdot|$ et $Q$. Pour ce faire, on fixe un élément $\omega \in \mathscr{H}_{0}$ et on va construire une primitive particulière dont la norme $L^{2}$ majorera $|\omega|_{\varepsilon}$.

On note $\bar{\varphi}$ la $p$-forme définie par $\bar{\varphi}=\varphi_{\omega}$ sur $\bar{U}$, et prolongée harmoniquement (pour la métrique $g$ ) sur $M$. On a alors $\|\bar{\varphi}\|_{H^{1}(M \backslash U)} \leq C\left\|\varphi_{\mid \partial U}\right\|_{H^{\frac{1}{2}(\partial U)}}$, la constante $C$ étant indépendante de $\varphi$ (la démonstration est similaire au cas des fonctions, cf. [Ta96], ch. 5, prop. 1.7). Par définition de $\mathscr{H}_{0}$, la $(p+1)$-forme $\bar{\omega}_{\infty}=\mathrm{d} \bar{\varphi}-\omega$ est un élément de $\mathscr{H}_{\infty}$ et elle est orthogonale à $\omega$. Elle vérifie donc $\left\|\bar{\omega}_{\infty}\right\|_{L^{2}(M \backslash U)}^{2} \leq\|\mathrm{d} \bar{\varphi}\|_{L^{2}(M \backslash U)}^{2} \leq\|\bar{\varphi}\|_{H^{1}(M \backslash U)}^{2}$ et elle admet une primitive $\bar{\varphi}_{\infty}$ nulle sur $U$ dont la norme vérifie $\left\|\bar{\varphi}_{\infty}\right\|^{2} \leq\left\|\bar{\omega}_{\infty}\right\|^{2} / \lambda^{(\mathrm{D})}$. Si on pose $\varphi=\bar{\varphi}-\bar{\varphi}_{\infty}$, on a alors $\mathrm{d} \varphi=\omega$ et

$$
\begin{aligned}
\|\varphi\|_{L^{2}(M \backslash U)} & \leq\|\bar{\varphi}\|_{L^{2}(M \backslash U)}+\left\|\bar{\varphi}_{\infty}\right\|_{L^{2}(M \backslash U)} \\
& \leq\|\bar{\varphi}\|_{L^{2}(M \backslash U)}+\|\bar{\varphi}\|_{H^{1}(M \backslash U)} / \lambda^{(\mathrm{D})^{1 / 2}},
\end{aligned}
$$

Toutes les normes étant ici relative à la métrique $g$.

Comme $\|\bar{\varphi}\|_{H^{1}(M \backslash U)} \leq C\left\|\varphi_{\mid \partial U}\right\|_{H^{\frac{1}{2}(\partial U)}}$ et que la norme $\left\|\varphi_{\mid \partial U}\right\|_{H^{\frac{1}{2}(\partial U)}}$ est elle-même contrôlée par la norme $H^{1}$ de $\varphi$ sur $U$, on a finalement

$$
\int_{M \backslash U}|\varphi|^{2} \mathrm{~d} v_{g} \leq C^{\prime}\|\varphi\|_{H^{1}(U)},
$$

où $C^{\prime}$ est une constante dépendant de $g$ mais pas de $\varepsilon$.

En utilisant le fait que $\varphi$ est cofermée sur $U$ (car égale à $\varphi_{\omega}$ ) et tangentielle le long de $\partial U$, une inégalité elliptique à trace associée à l'opérateur $\mathrm{d}+\delta$ (voir [Ta96], section 5.9) donne

$$
\|\varphi\|_{H^{1}(U)} \leq C^{\prime \prime}\left(\|\varphi\|_{L^{2}(U)}+\|\mathrm{d} \varphi\|_{L^{2}(U)}\right)=C^{\prime \prime}\left(|\omega|+Q(\omega)^{\frac{1}{2}}\right),
$$

la métrique considérée étant ici encore $g$.

Pour une métrique $g_{\varepsilon}$, on a $|\omega|_{\varepsilon}^{2} \leq\|\varphi\|_{L^{2}(U)}^{2}+\varepsilon^{n-2 p}\|\varphi\|_{L^{2}(M \backslash U)}^{2}$. Comme $\|\varphi\|_{L^{2}(U)}=|\omega|$ et que $\|\varphi\|_{L^{2}(M \backslash U)}$ peut être majoré à l'aide de (1) et (2), on obtient la majoration

$$
|\omega|_{\varepsilon}^{2} \leq|\omega|^{2}+\varepsilon^{n-2 p} C^{\prime} C^{\prime \prime 2}\left(|\omega|+Q(\omega)^{\frac{1}{2}}\right)^{2}
$$

qui permet d'appliquer la remarque 18 et le lemme 17.

\section{Prescription du spectre}

Pour construire des valeurs propres multiples nous allons nous utiliser, outre théorème de convergence spectrale 11, une propriété de transversalité vérifiée par des valeurs 
propres multiples sur des espaces modèles. Cette propriété remonte à Arnol'd et a été précisée par Y. Colin de Verdière dans [CdV88], nous allons en rappeler une définition :

On suppose qu'on a une famille d'opérateurs $\left(P_{a}\right)_{a \in B^{k}}$, où $B^{k}$ est la boule unité de $\mathbb{R}^{k}$ (en pratique, $P_{a}$ est le laplacien associé à une métrique $g_{a}$ ), tels que $P_{0}$ possède une valeur propre $\lambda_{0}$ d'espace propre $E_{0}$ et de multiplicité $N$. Pour les petites valeurs de $a, P_{a}$ possède des valeurs propres proches de $\lambda_{0}$ dont la somme des espaces propres est de dimension $N$. Comme dans la définition de l'écart spectral, on identifie cette somme à $E_{0}$ et on note $q_{a}$ la forme quadratique associée à $P_{a}$ transportée sur $E_{0}$.

Définition 20. On dit que $\lambda_{0}$ vérifie l'hypothèse de transversalité d'Arnol'd si l'application $\Psi: a \mapsto q_{a}$ de $B^{k}$ dans $Q\left(E_{0}\right)$ est essentielle en 0 , c'est-à-dire qu'il existe $\varepsilon>0$ tel que si $\Phi: B^{k} \rightarrow \mathcal{Q}\left(E_{0}\right)$ vérifie $\|\Psi-\Phi\|_{\infty} \leq \varepsilon$, alors il existe $a_{0} \in B^{k}$ tel que $\Phi\left(a_{0}\right)=q_{0}$.

Une propriété cruciale est que si $\Phi$ provient d'une famille $\left(P_{a}^{\prime}\right)$ d'opérateurs, alors $\lambda_{0}$ est valeur propre de $P_{a_{0}}^{\prime}$ de multiplicité $N$ et vérifie la même propriété de transversalité : on dit que cette valeur propre multiple est stable. Comme remarqué dans [CdV88], on peut généraliser cette définition à une suite finie de valeur propre.

Pour démontrer les théorèmes 1 et 4 , nous allons construire des domaines modèles dont le début du spectre vérifie la propriété de stabilité pour ensuite appliquer le théorème 11. Ces domaines seront des produits d'une sphère et d'une boule, ils pourront donc être plongé dans n'importe quelle variété de même dimension.

La multiplicité stable sur un produit sera obtenue grâce à la formule de Künneth : si $\left(M_{1}, g_{1}\right)$ et $\left(M_{2}, g_{2}\right)$ sont deux variétés riemanniennes compactes et $\alpha_{i} \in \Omega^{*}\left(M_{i}\right)$, $i=1,2$, alors on a, en identifiant chacune des formes $\alpha_{i}$ à son relevé sur $\left(M_{1} \times\right.$ $\left.M_{2}, g_{1} \oplus g_{2}\right)$,

$$
\Delta\left(\alpha_{1} \wedge \alpha_{2}\right)=\Delta \alpha_{1} \wedge \alpha_{2}+\alpha_{1} \wedge \Delta \alpha_{2}
$$

En particulier, si $\alpha_{i}$ est une forme propre de valeur propre $\lambda_{i}$ pour $i=1,2$, alors $\alpha_{1} \wedge \alpha_{2}$ est une forme propre de valeur propre $\lambda_{1}+\lambda_{2}$ pour la métrique produit. Il est clair que que si $\alpha_{1}$ et $\alpha_{2}$ sont fermées, alors $\alpha_{1} \wedge \alpha_{2}$ aussi. On peut vérifier que pour la métrique produit, si $\alpha_{1}$ et $\alpha_{2}$ sont cofermées (par exemple si l'une des deux est une fonction) leur produit est aussi cofermé.

On va montrer que si l'une des deux valeurs propres est de multiplicité stable, la valeur propre multiple obtenue sur la variété produit peut hériter de cette propriété.

Lemme 21. On suppose que $\mu_{p_{1}, k_{1}}\left(M_{1}, g_{1}\right)$ est une valeur propre simple, $\mu_{p_{2}, k_{2}}\left(M_{2}, g_{2}\right)$ une valeur propre de multiplicité stable $N$ et qu'il n'y a pas d'autres valeurs propres de $M_{1}$ et $M_{2}$ dont la somme soit $\mu_{p_{1}, k_{1}}\left(M_{1}, g_{1}\right)+\mu_{p_{2}, k_{2}}\left(M_{2}, g_{2}\right)$. Alors $\mu_{p_{1}, k_{1}}\left(M_{1}, g_{1}\right)+\mu_{p_{2}, k_{2}}\left(M_{2}, g_{2}\right)$ est valeur propre de multiplicité stable $N$ $\operatorname{sur}\left(M_{1} \times M_{2}, g_{1} \oplus g_{2}\right)$. 
Démonstration. Si on note $\alpha$ une forme propre de $\mu_{p_{1}, k_{1}}\left(M_{1}, g_{1}\right)$ et $E$ l'espace propre de $\mu_{p_{2}, k_{2}}\left(M_{2}, g_{2}\right)$ alors, en vertu des remarques qui précèdent, $\alpha \wedge E$ est un espace propre cofermé de valeur propre $\mu_{p_{1}, k_{1}}\left(M_{1}, g_{1}\right)+\mu_{p_{2}, k_{2}}\left(M_{2}, g_{2}\right)$.

Supposons que $\mu_{p_{2}, k_{2}}\left(M_{2}, g_{2}\right)$ vérifie la propriété 20 pour la famille de métrique $g_{2, a}$ et la famille de forme quadratique $q_{a}$. Si la forme $\alpha$ est normée, le produit par $\alpha$ plonge isométriquement $\Omega\left(M_{2}\right)$ dans $\Omega\left(M_{1} \times M_{2}\right)$ en envoyant espace propre sur espace propre, quel que soit $a$. En particulier, les espaces propres de $\left(M_{2}, g_{2, a}\right)$ de valeur propre proche de $\mu_{p_{2}, k_{2}}\left(M_{2}, g_{2}\right)$, sont envoyé sur des espaces propres de même multiplicité, leur valeur propre étant augmenté de $\mu_{p_{1}, k_{1}}\left(M_{1}, g_{1}\right)$. L'espace $\alpha \wedge E$ vérifie donc de la propriété 20 pour la valeur propre $\mu_{p_{1}, k_{1}}\left(M_{1}, g_{1}\right)+\mu_{p_{2}, k_{2}}\left(M_{2}, g_{2}\right)$ et la famille de forme quadratique $q_{a}+\mu_{p_{1}, k_{1}}\left(M_{1}, g_{1}\right)|\cdot|^{2}$.

Nous utiliserons plusieurs fois le fait que sur une boule euclidienne de rayon $\varepsilon$, le noyau du laplacien pour la condition de bord (A) est trivial, sauf en degré 0 pour lequel sa dimension est 1 . Toutes les autres valeurs propres tendent vers $+\infty$ quand $\varepsilon \rightarrow 0$. En particulier, sur un produit riemannien $M \times B^{k}(\varepsilon)$, les premières formes propres sont les relevés des premières formes propres de $M$.

Les trois lemmes qui suivent ont pour but de construire les domaines modèles :

Lemme 22. Pour tous entiers $N \geq 1, n \geq 3$, toute suite finie $0<a_{1} \leq a_{2} \leq \cdots \leq$ $a_{N}$ et toute constante $C>a_{N}$, il existe une métrique $g$ sur $S^{n}$ telle que $\mu_{0, i}=a_{i}$ pour $i \leq N$, ces valeurs propres vérifiant l'hypothèse de stabilité, $\mu_{0, N+1}>C$ et $\mu_{p, 1}>C$ pour $1 \leq p \leq\left[\frac{n-1}{2}\right]$.

Démonstration. Le résultat sur les $\mu_{0, i}$ découle des travaux de Colin de Verdière (voir [CdV86] et [CdV87]), il suffit de montrer que la construction géométrique peut se faire avec $\mu_{p, 1}>C$ pour $p \geq 1$. Le principe de la démonstration est d'appliquer le théorème 11 avec un domaine $U$ dont le spectre vérifie la conclusion du lemme. Pour appliquer l'argument de stabilité, on ne doit pas travailler avec une seule métrique sur $U$ mais une petite famille de métriques, un élément crucial sera alors que l'invariant conforme qui minore le spectre en degré $\left[\frac{n}{2}\right]$ varie continûment pour la topologie $C^{0}$ (voir remarque 14), il sera donc uniformément minoré pour cette famille de métrique.

On procède par récurrence sur la dimension. En dimension 3 et 4 , on procède comme dans [CdV87] : On choisit une surface $\Sigma$ dont le début du spectre (pour les fonctions) est égal à $a_{1} \leq a_{2} \leq \cdots \leq a_{N}$ et vérifie l'hypothèse de stabilité, et on choisit comme domaine $U$ le produit riemannien de $\Sigma$ avec un petit intervalle ] $-\varepsilon, \varepsilon$ [ (en dimension 3) ou un petit disque de rayon $\varepsilon$ (en dimension 4), la métrique étant prolongée de manière quelconque en dehors de $U$. Le théorème 11 et l'argument de stabilité assure l'existence d'une métrique $g$ telle que $\mu_{0, i}=a_{i}$ pour $i \leq N$, et $\mu_{0, N+1}>C$, et comme son volume est arbitrairement petit, le théorème 13 et la remarque 14 assurent qu'on peut choisir $g$ telle qu'on ait aussi $\mu_{1,1}>C$. 
$\mathrm{Si}$, par hypothèse de récurrence, le lemme est vrai en dimension $n-1$, on raisonne comme en dimension 3 et 4 en munissant $S^{n-1}$ de la métrique fournie par le lemme et en utilisant le domaine $\left.U=S^{n-1} \times\right]-\varepsilon, \varepsilon$ [ plongé dans $S^{n}$. Le théorème 11 donne une métrique $S^{n}$ qui a le spectre souhaité pour $p \leq\left[\frac{n-3}{2}\right]\left(H^{p}(U / M)\right.$ étant trivial), et le théorème 13 assure que $\mu_{\left[\frac{n-1}{2}\right], 1}>C$.

Lemme 23. Pour tous entiers $N \geq 1, p \geq 2$ et $n \geq 2 p+3$, toute suite finie $0<a_{1} \leq a_{2} \leq \cdots \leq a_{N}$ et toute constante $C>a_{N}$, il existe une métrique $g$ sur la variété $M^{n}=S^{p+1} \times B^{p+2}$ pour $n=2 p+3$ ou $M^{n}=S^{n-1} \times[0,1]$ si $n>2 p+3$, telle que $\mu_{p, i}=a_{i}$ pour $i \leq N$, ces valeurs propres vérifiant l'hypothèse de stabilité, $\mu_{p, N+1}>C$ et $\mu_{q, 1}>C$ pour $1 \leq q \leq \frac{n}{2}, q \neq p$, le volume $\operatorname{Vol}(M, g)$ étant arbitrairement petit.

Démonstration. Remarquons d'abord qu'en prescrivant le début du spectre pour $p=$ 0 dans le lemme 22, on a aussi prescrit les $\mu_{p, i}$ pour $p=n-1$ et $i \leq N$, par dualité de Hodge. Partant de ce constat, on va encore procéder par récurrence sur $n$, le degré $p$ étant fixé.

Pour $n=2 p+3$, il suffit de considérer $M=S^{p+1} \times B^{p+2}(\varepsilon)$, la sphère $S^{p+1}$ étant munie de la métrique fournie par le lemme 22 et $B^{p+2}(\varepsilon)$ étant une boule de rayon $\varepsilon$ petit.

Pour $n>2 p+3$, la récurrence s'effectue comme dans le lemme 22 : on applique le théorème 11 au domaine $M^{n-1}$ plongé dans $S^{n-1}$ et on pose $\left.M^{n}=S^{n-1} \times\right]-\varepsilon, \varepsilon[$.

Lemme 24. Pour tous entiers $N \geq 1, n \geq 3$, toute suite finie $0<a_{1}<a_{2} \leq a_{3} \leq$ $\cdots \leq a_{N}$ et toute constante $C>a_{N}$, il existe une métrique $g$ sur $M=B^{3} \times S^{n}$ telle que $\mu_{1, i}(M, g)=a_{i}$ pour $i \leq N$ (avec stabilité), $\mu_{1, N+1}(M, g)>C$ et $\mu_{p, 1}(M, g)>C$ pour $2 \leq p \leq \frac{n}{2}$, le volume $\operatorname{Vol}(M, g)$ étant arbitrairement petit.

Démonstration. On va une nouvelle fois utiliser le théorème 11 avec un domaine $U$ produit d'une sphère et d'une boule, mais avec une métrique particulière sur la boule : selon [Gu04] (théorème 2.1), il existe une métrique $g_{B}$ sur $B^{3}$ telle que $\mu_{1,1}\left(B^{3}, g_{B}\right)=a_{1}, \mu_{1,2}\left(B^{3}, g_{B}\right)>C$ et $\mu_{p, 1}\left(B^{3}, g_{B}\right)>C$ pour $p=0,2$, le volume étant arbitrairement petit. En utilisant le lemme 22, on peut munir $S^{n}$ d'une métrique telle que $\mu_{0, i}\left(S^{n}\right)=a_{i}-a_{1}$ pour $i \leq N-1, \mu_{0, N}\left(S^{n}\right)>C$ et $\mu_{p, 1}\left(S^{n}\right)>C$ pour $p \geq 1$. Le produit $M=S^{n} \times B^{3}$ vérifie alors $\mu_{1, i}(M)=$ $\mu_{0, i}\left(S^{n}\right)+\mu_{1,1}\left(B^{3}, g_{B}\right)=a_{i}$ pour $i \leq N, \mu_{1, N+1}(M)>C$ et $\mu_{p, 1}(M)>C$ pour $2 \leq p \leq \frac{n}{2}$.

On est maintenant en mesure de démontrer les résultats annoncés dans l'introduction. 
Demonstration du théorème 1 . Pour chaque degré $p$, on se donne le domaine $U_{p}$ fourni par le lemme 23 (ou le lemme 24 pour $p=1$ ) dont le début du spectre en degré $p$ est celui qu' on veut prescrire, avec $C>\sup _{p, i} a_{p, i}$. On peut noter que dans la démonstration du théorème 11 , l'hypothèse de connexité du domaine $U$ n'intervient pas, la conclusion reste donc vraie en remplaçant $U$ par un nombre fini de domaine. On peut donc l'appliquer à la famille $\left(U_{p}\right)$ plongée dans $M$ (en remarquant que $H^{p}\left(\left(\cup U_{p}\right) / M\right)$ est trivial pour $\left.1 \leq p \leq \frac{n-3}{2}\right)$, et grâce à la stabilité du spectre de ces domaines on conclut à l'existence d'une métrique $g$ sur $M$ telle que $\mu_{p, k}(M, g)=$ $a_{p, k}$ pour pour $1 \leq k \leq N$ et $1 \leq p \leq\left[\frac{n-3}{2}\right]$. Comme pour les lemmes précédents, le cas du degré $p=\left[\frac{n-1}{2}\right]$ est couvert par le théorème 13 .

Pour montrer qu' on peut aussi prescrire le volume, on procède comme dans [Gu04] et [Ja08] : on peut appliquer l'argument de stabilité au volume en le traitant comme une valeur propre simple. Il suffit donc d'ajouter à la famille $U_{p}$ une boule de volume $V-\sum_{p} \operatorname{Vol}\left(U_{p}\right)$ (en ayant choisit les volumes des $U_{p}$ suffisamment petits) et dont les valeurs propres pour les $p$-formes sont arbitrairement grandes (c'est possible selon [GP95]). Le fait que le volume de cette boule vérifie l'hypothèse de transversalité signifie simplement qu'on peut lui donner n'importe quelle valeur au voisinage de $V-\sum_{p} \operatorname{Vol}\left(U_{p}\right)$, par exemple par homothétie.

Demonstration du théorème 4. Y. Colin de Verdière a montré que la première valeur propre de la sphère $S^{2}$ muni de sa métrique canonique est stable ([CdV88], section 2). On peut donc raisonner comme dans le lemme 23 : la valeur propre $\mu_{1,1}\left(S^{2}\right)$ est de multiplicité 3 stable et il suffit d'appliquer le théorème 11 au domaine $S^{2} \times B^{n-2}(\varepsilon)$.

\section{Références}

[Am03] B. Ammann, A spin-conformal lower bound of the first positive Dirac eigenvalue. Differential Geom. Appl. 18 (2003), no. 1, 21-32. Zbl 1030.58020 MR 1951070

[An89] C. Anné, Principe de Dirichlet pour les formes différentielles. Bull. Soc. Math. France 117 (1989), (4), 445-450. Zbl 0703.53033 MR 1042432

[BCC98] G. Besson, B. Colbois et G. Courtois, Sur la multiplicité de la première valeur propre de l'opérateur de Schrödinger avec champ magnétique sur la sphère $S^{2}$. Trans. Amer. Math. Soc. 350 (1998), (1), 331-345. Zbl 0903.58060 MR 1390969

[BD97] G. Baker et J. Dodziuk, Stability of spectra of Hodge-de Rham laplacians. Math. Z. 224 (1997), no. 3, 327-345,. Zbl 1004.58016 MR 1439194

[Be80] G. Besson, Sur la multiplicité de la première valeur propre des surfaces riemanniennes. Ann. Inst. Fourier 30 (1980), no. 1, 109-128. Zbl 0417.30033 MR 0576075

[CdV86] Y. Colin de Verdière, Sur la multiplicité de la première valeur propre non nulle du laplacien. Comment. Math. Helv. 61 (1986), no. 2, 254-270. Zbl 0607.53028 MR 0856089 
Vol. 86 (2011) Sur la multiplicité des valeurs propres du laplacien de Hodge-de Rham 983

[CdV87] Y. Colin de Verdière, Construction de laplaciens dont une partie finie du spectre est donnée. Ann. Sci. École Norm. Sup. 20 (1987), no. 4, 599-615. Zbl 0636.58036 MR 0932800

[CdV88] Y. Colin de Verdière, Sur une hypothèse de transversalité d'Arnol'd. Comment. Math. Helv. 63 (1988), no. 2, 184-193. Zbl 0672.58046 MR 0948776

[CdVT93] Y. Colin de Verdière et N. Torki, Opérateur de Schrödinger avec champ magnétique. In Séminaire de théorie spectrale et géométrie, Semin. Theor. Spectr. Geom. 11, Institut Fourier, Saint-Martin-d'Hères 1993, 9-18. Zbl 0937.35510 MR 1715941

[Ch76] S. Y. Cheng, Eigenfunctions and nodal sets. Comment. Math. Helv. 51 (1976), no. 1, 43-55. Zbl 0334.35022 MR 0397805

[Ch79] J. Cheeger, Analytic torsion and the heat equation. Ann. of Math. 109 (1979), no. 2, 259-322. Zbl 0412.58026 MR 0528965

[Co04] B. Colbois, Spectre conforme et métriques extrémales. In Séminaire de théorie spectrale et géométrie, Semin. Theor. Spectr. Geom. 22, Institut Fourier, Saint-Martind'Hères, 2004, 93-101. Zbl 1069.58017 MR 2136138

[Da05] M. Dahl, Prescribing eigenvalues of the Dirac operator. Manuscripta Math. 118 (2005), no. 2, 191-199. Zbl 1081.58021 MR 2177685

[Do82] J. Dodziuk, Eigenvalues of the Laplacian on forms. Proc. Amer. Math. Soc. 85 (1982), 437-443. Zbl 0502.58038 MR 0656119

[Er02] L. Erdős, Spectral shift and multiplicity of the first eigenvalue of the magnetic Schrödinger operator in two dimensions. Ann. Inst. Fourier 52 (2002), no. 6, 18331874. Zbl MR

[GP95] G. Gentile et V. Pagliara, Riemannian metrics with large first eigenvalue on forms of degree p. Proc. Amer. Math. Soc. 123 (1995), no. 12, 3855-3858. Zbl 0848.53022 MR 1277111

[Gu04] P. Guérini, Prescription du spectre du laplacien de Hodge-de Rham. Ann. Sci. École Norm. Sup. 37 (2004), no. 2, 270-303. Zbl 1068.58016 MR 2061782

[HHN99] M. Hoffmann-Ostenhof, T. Hoffmann-Ostenhof et N. Nadirashvili, On the multiplicity of eigenvalues of the Laplacian on surfaces. Ann. Global Anal. Geom. 17 (1999), no. 1, 43-48. Zbl 0923.35109 MR 1674331

[Ja07] P. Jammes, Minoration conforme du spectre du laplacien de Hodge-de Rham. Manuscripta Math. 123 (2007), no. 1, 15-23. Zbl 1127.35027 MR 2300056

[Ja08] P. Jammes, Prescription du spectre du laplacien de Hodge-de Rham dans une classe conforme. Comment. Math. Helv. 83 (2008), no. 3, 521-537. Zbl 1155.58305 MR 2410778

[Ja09a] P. Jammes, Construction de valeurs propres doubles du laplacien de Hodge-de Rham. J. Geom. Anal. 19 (2009), no. 3, 643-654. Zbl 1173.58011 MR 2496570

[Ja09b] P. Jammes, Sur la multiplicité des valeurs propres d'une variété compacte. In Actes du séminaire de théorie spectrale et géométrie, Semin. Theor. Spectr. Geom. 26, Institut Fourier, Saint-Martin-d'Hères, 2009, 1-11. Zbl 05666393 MR 2654595

[Lo96] J. Lohkamp, Discontinuity of geometric expansions. Comment. Math. Helv. 71 (1996), no. 2, 213-228. Zbl 0857.58041 MR 1396673 
[Mc93] J. McGowan, The $p$-spectrum of the Laplacian on compact hyperbolic three manifolds. Math. Ann. 297 (1993), no. 4, 725-745. Zbl 0801.53034 MR 1245416

[Na88] N. Nadirashvili, Multiple eigenvalues of the Laplace operator. Math. USSR-Sb. 61 (1988), no. 1, 225-238. Zbl 0672.35049 MR 0905007

[Sé94] B. Sévennec, Multiplicité du spectre des surfaces : une approche topologique. In Séminaire de théorie spectrale et géométrie, Semin. Theor. Spectr. Geom. 12, Institut Fourier, Saint-Martin-d'Hères, 1994, 29-36. Zbl 0909.58059 MR 1714546

[Sé02] B. Sévennec, Multiplicity of the second Schrödinger eigenvalue on closed surfaces. Math. Ann. 324 (2002), no. 1, 195-211. Zbl 1053.58014 MR 1931764

[Ta96] M. Taylor, Partial differential equations I. Appl. Math. Sci. 115, Springer-Verlag, New York 1996. Zbl 0869.35002 MR 1395148

Received April 29, 2009

Pierre Jammes, Laboratoire J.-A. Dieudonné, Université Nice - Sophia Antipolis, Parc Valrose, 06108 Nice Cedex 02, France

E-mail: pjammes@unice.fr 